\title{
Use of chimerism analysis after allogeneic stem cell transplantation: Belgian guidelines and review of the current literature
}

Anke Delie, Anke Verlinden, Karolien Beel, Dries Deeren, Dominiek Mazure, Frédéric Baron, Dimitri Breems, Ann De Becker, Carlos Graux, Philippe Lewalle, Johan Maertens, Xavier Poire, Helene Schoemans, Dominik Selleslag, Florence Van Obbergh \& Tessa Kerre

To cite this article: Anke Delie, Anke Verlinden, Karolien Beel, Dries Deeren, Dominiek Mazure, Frédéric Baron, Dimitri Breems, Ann De Becker, Carlos Graux, Philippe Lewalle, Johan Maertens, Xavier Poire, Helene Schoemans, Dominik Selleslag, Florence Van Obbergh \& Tessa Kerre (2020): Use of chimerism analysis after allogeneic stem cell transplantation: Belgian guidelines and review of the current literature, Acta Clinica Belgica, DOI: 10.1080/17843286.2020.1754635

To link to this article: https://doi.org/10.1080/17843286.2020.1754635

\section{Published online: 02 May 2020.}

Submit your article to this journal $\pi$

щ Article views: 90

Q View related articles ¿

View Crossmark data $[\pi$ 


\title{
Use of chimerism analysis after allogeneic stem cell transplantation: Belgian guidelines and review of the current literature
}

\author{
Anke Delie ${ }^{a}$, Anke Verlinden ${ }^{b}$, Karolien Beelc, Dries Deeren ${ }^{d}$, Dominiek Mazure ${ }^{a}$, Frédéric Barone, \\ Dimitri Breems'c, Ann De Beckerf, Carlos Graux', Philippe Lewalleh, Johan Maertensi, Xavier Poirej, \\ Helene Schoemansi, Dominik Selleslagk', Florence Van Obbergh' and Tessa Kerrea*
}

\begin{abstract}
aDepartment of Hematology, University Hospital, Ghent University, Ghent, Belgium; 'Department of Hematology, University Hospital, University of Antwerp, Antwerp, Belgium; 'Department of Hematology, Ziekenhuis Netwerk, Antwerpen, Belgium; ${ }^{\mathrm{d} D e p a r t m e n t}$ of Hematology, AZ Delta, Roeselare, Belgium; 'Department of Hematology, University Hospital, University of Liège, Liège, Belgium; fDepartment of Hematology, University Hospital, Vrije Universiteit Brussel, Brussels, Belgium; 'Department of Hematology, University

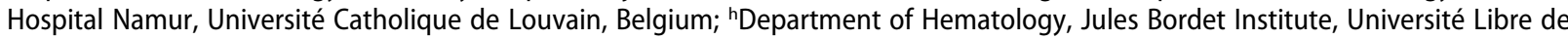
Bruxelles, Brussels, Belgium; 'Department of Hematology, University Hospital, KU Leuven, Leuven, Belgium; 'Department of Hematology, University Hospital Saint Luc, Univeristé Catholique de Louvain, Brussels, Belgium; kDepartment of Hematology, AZ Sint-Jan, Bruges, Belgium; 'Department of Hematology, CH Jolimont, La Louvièvre, Belgium
\end{abstract}

\section{ABSTRACT}

Background: Allogeneic hematopoietic stem cell transplantation (HSCT) is a curative treatment option in both adult and pediatric patients with malignant and non-malignant hematological diseases. Chimerism analysis, which determines the donor or recipient origin of hematopoietic cells in HSCT recipients, is an essential aspect of post-HSCT follow-up.

Objectives: To review the current literature and develop Belgian consensus guidelines for the use of chimerism analysis in the standard of care after allogeneic HSCT.

Methods: Non-systematic review of the literature in consultancy with the members of the BHS transplantation committee.

Results: Clinical application with regards to prediction of graft failure or relapse as well as cell source are reviewed. A consensus guideline on the use of chimerism analysis after HSCT is presented.

Conclusion: Monitoring of the dynamics or kinetics of a patient's chimerism status by serial analysis at fixed time points, as well as on suspicion of relapse or graft failure, is needed to monitor engraftment levels, as well as disease control and possible relapse.

\section{KEYWORDS}

Allogeneic stem cell transplantation; chimerism; minimal residual disease; graft failure

\section{Introduction}

Allogeneic hematopoietic stem cell transplantation (HSCT) is a curative treatment option in both adult and pediatric patients with malignant and non-malignant hematological diseases. The introduction of reduced intensity conditioning (RIC) regimens as well as advances in donor selection and supportive care have led to a reduction in the treatment-related mortality associated with allogeneic HSCT, allowing an increasing number of patients to benefit from this treatment. In Belgium, 641 allogeneic HSCTs were performed between 1/1/2016 and 31/12/2017, of which 412 (64\%) RIC HSCTs.

However, disease relapse and secondary graft failure remain an important cause of treatment failure post allogeneic HSCT. Early detection of relapse and monitoring of engraftment levels are an essential aspect of post-HSCT follow-up. The level of engraftment can be monitored post-HSCT by determining the genotype of origin of hematopoietic cells, or chimerism analysis. This allows for the detection of persistent or recurrent recipient hematopoiesis, which can be used as a surrogate marker for disease relapse. Also, decreasing donor chimerism can be the first sign of graft failure.

Chimerism analysis can be performed on unfractionated peripheral blood cells, bone marrow or isolated cell lineages. A patient can either have a complete chimerism (CC), when the \% of donor origin cells exceeds the upper detection limit of the analysis used (usually corresponding with donor chimerism of $>95-99.9 \%$ depending on the sensitivity of the used test cfr. infra), or a mixed chimerism (MC) where donor and recipient hematopoietic cells coexist. However, the state of a patient's chimerism is subject to change over time and a patient can present with an increasing or decreasing donor chimerism, i.e. an increasing or decreasing amount of donor hematopoiesis compared to previous analysis. Lastly, levels of chimerism are not necessarily

CONTACT Anke Delie anke.delie@uzgent.be Department of Hematology, University Hospital, Ghent University, C. Heymanslaan 10, Gent 9000, Belgium

*On behalf of the BHS transplantation committee.

Members of the BHS transplantation committee: Tessa Kerre, Frédéric Baron, Karolien Beel, Zwi Berneman, Marleen Bogaert, Dimirti Breems, Ann De Becker, Dries Deeren, Yves Beguin, Dorien Deneve, Carlos Graux, Julie Herman, Audrey Janssen, Philippe Lewalle, Tom Lodewyck, Johan Maertens, Dominiek Mazure, Xavier Poiré, Helene Schoemans, Rik Schots, Dominik Selleslag, Sophie Servais, Florence Van Obbergh, Anke Verlinden, Evelyne Willems, Sebastian Wittnebel, Pierre Zachée, Marleen Bogaert, Koen Theunissen and Marielle Beckers.

C 2020 Journal of Belgian Society of Internal Medicine and Royal Belgian Society of Laboratory Medicine 
Table 1. Definitions of chimerism and engraftment.

\begin{tabular}{|c|c|c|}
\hline Chimerism state & Definition & Comment \\
\hline Complete chimerism (CC) & All hematopoietic cells are of donor origin [1] & $\begin{array}{l}\text { Usually defined as }>95-99.9 \% \text { donor origin of hematopoietic cells } \\
\text { depending on test sensitivity ( } \%>\text { detection limit for chosen } \\
\text { analysis) }\end{array}$ \\
\hline Mixed chimerism (MC) & $\begin{array}{l}\text { Presence of both donor and recipient } \\
\text { hematopoietic cells [1] }\end{array}$ & \\
\hline Split chimerism & $\begin{array}{l}\text { CC has been achieved in one or more cell lineage } \\
\text { but MC still exists in other cell lineages [1] }\end{array}$ & \\
\hline Engraftment & $\begin{array}{l}\text { First of } 3 \text { consecutive days with absolute } \\
\text { neutrophil count (ANC) }>500 / \mu \mathrm{L} \text { (of donor } \\
\text { origin) [6] }\end{array}$ & $\begin{array}{l}\text { Platelet engraftment: sustained platelet count }>20.000 / \mu \mathrm{L} \text { for } 7 \text { days } \\
\text { without transfusion [6] }\end{array}$ \\
\hline Primary graft failure & $\begin{array}{l}\text { Absence of engraftment/hematological recovery } \\
\text { with donor cells in the first month post-HSCT [6] }\end{array}$ & $\begin{array}{l}\text { ANC }<500 / \mu \mathrm{L} \mathrm{d}+28[6] \\
\text { In case of RIC or NMA HSCT autologous recovery of ANC is possible, } \\
\text { confirmation of graft failure by chimerism analysis is required [6] }\end{array}$ \\
\hline Secondary graft failure & $\begin{array}{l}\text { Loss of graft function }(\mathrm{ANC}<500 / \mu \mathrm{L}) \text { after initial } \\
\text { engraftment }[6]\end{array}$ & $\begin{array}{l}\text { Donor chimerism }>5 \% \\
\text { Without secondary cause such as relapse, infection, marrow } \\
\text { toxicity...[6] }\end{array}$ \\
\hline Autologous recovery & $100 \%$ recipient origin of hematopoietic cells [1] & \\
\hline
\end{tabular}

Table 2. Sensitivity and informativity* of different chimerism detection methods.

\begin{tabular}{|c|c|c|}
\hline $\begin{array}{l}\text { Detection } \\
\text { method }\end{array}$ & Sensitivity & Informativity* \\
\hline X/Y FISH & $0,001-0,1 \%$ & $\begin{array}{c} \pm 50 \% \text { - only applicable in sex } \\
\text { mismatched HSCT }\end{array}$ \\
\hline STR-PCR & $1-5 \%$ & (nearly) $100 \%$ \\
\hline Rt-qPCR & $0,01-0,1 \%$ & $>90 \%$ \\
\hline
\end{tabular}

*The informativity or applicability of the test refers to the proportion of donor/recipient couples that can be evaluated using this technique.

the same between different cell populations and patients can present with a split chimerism, where CC has been achieved in one or more cell lineages but MC still exists in other cell lineages [1-5]. (Table 1)

Analysis of a patient's chimerism status is based on the identification of the genetic profile of the patient and his or her donor in the recipient's blood or bone marrow. This is accomplished by the analysis of differences in polymorphic genetic markers such as short tandem repeats (STR), single nucleotide polymorphisms (SNP) or insertions and deletions between donor and recipient $[1,2,4,7,8]$. Both recipient and donor pre-transplant DNA samples need to be available for genotyping to establish pre-transplant genetic profiles [2]. Ideally, whole blood samples should be provided, but cheek swabs can be used as an alternative if peripheral blood counts are too low.

Short tandem repeats are units of 2 to 6 base pairs in length repeated multiple times within a given DNA fragment. These DNA fragments have a highly polymorphic nature between individuals, with a variable number of repeats at each tested locus. By using PCRbased amplification of these loci, patient and donor DNA can be identified based on the length of the resulting PCR products [2,9-12]. There are several commercial kits available with up to 16 STR markers, originally designed for use in forensic identification, which cover multiple ethnic backgrounds [2,9,12]. Short tandem repeat based chimerism analysis has a reported sensitivity of $1-5 \%[1,10,11,13]$ with a high reproducibility and an applicability (or informativity, i.e. the number of donor/recipient couples that can be evaluated using this technique) of nearly $100 \%$ [14]. (Table 2)

Since the early 2000's real time quantitative PCR (rtqPCR) analysis of SNPs or insertions and deletions is also possible $[1,2,4,7,8]$. These analyses are based on real time amplification of known SNPs or insertion/deletion polymorphisms, for which several commercial kits are available $[1,9,10,14,15]$. This technique has a reported sensitivity of $0.01-0.1 \%$ [14-16] and a high degree of informativity of $>90 \%[9,14]$. (Table 2) Various studies have shown a high concordance rate between STRbased methods and $\mathrm{qPCR}$, the described discordant results have all been in line with the reported differences in sensitivity between both analyses (with rt-qPCR detecting MC earlier than STR-based techniques) [9,10,16-19].

Novel techniques using digital droplet PCR methods are in development but as of yet, have not been standardized for use outside clinical trials, as no standard testing panel exists $[4,20]$.

In sex mismatched HSCT X/Y FISH can be used for chimerism detection. This technique is well standardized, has shown a high sensitivity of $0,1-0,001 \%$ and can be rapidly performed in most laboratories. However, as it is restricted to sex mismatched HSCTs it has a lower clinical applicability (approximately $50 \%$ of patients receive a sex mismatched HSCT) $[1,21]$. (Table 2)

Semi quantitative fluorescent PCR of STRs remains the current gold standard for chimerism monitoring because of the high level of standardization, high applicability, reproducibility, costeffectiveness, short laboratory turnaround time and clinical validation in multiple follow-up studies $[4,9,22,23]$. Currently, it remains the most commonly used method for chimerism analysis in Belgium. In a survey carried out amongst 12 Belgian allotransplant centers, this technique was used in 11 out of the 12 centers routinely using chimerism analysis in post-HSCT follow-up. 


\section{Clinical application of chimerism analysis}

Monitoring the dynamics or kinetics of a patient's chimerism status by serial analysis at fixed time points, as well as on suspicion of relapse or graft failure, can be used to monitor engraftment levels, as well as disease control and possible relapse [9,24-26]. Chimerism kinetics post-HSCT varies greatly amongst patients and is influenced, amongst other factors, by the conditioning regimen [24,27-31].

The use of RIC and Non myeloablative (NMA) conditioning regimens has allowed older patients and patients with existing co-morbidities to proceed to allogeneic HSCT by reducing the treatment-related morbidity and mortality. These regimens do not rely on an immediate complete eradication of host hematopoiesis, but rather on the immunologic graft versus tumor (GVT) effect, allowing for a faster hematological recovery post-HSCT. However, MC is observed at the time of engraftment in nearly all patients who undergo NMA conditioning and a substantial subset of patients undergoing RIC transplantation [24,30]. In contrast, patients who receive a Myeloablative conditioning (MAC) regimen tend to reach $C C$ at an earlier time point post-HSCT, with the majority of patients reaching $\mathrm{CC}$ at day 30 , especially when using a TBI-based regimen $[5,29,32,33]$.

\section{Secondary graft failure}

The presence of MC (in both whole blood, bone marrow or T-cells) has been linked to the occurrence of secondary graft failure in both malignant and non-malignant disease, with a proportional increase of the risk of secondary graft failure with an increasing degree of MC [24,29,34,35]. Both Lee et al. [29] and Lucarelli et al. [34] showed that secondary graft failure mostly occurred in those patients with a MC of $<75 \%$ hematopoietic cells of donor origin. Baron et al. [24] showed that the presence of $<50 \%$ donor-derived T-cells or NK-cells on day 14 after non myeloablative (NMA) transplant, was associated with a higher risk of graft rejection.

In a cohort of 335 patients transplanted for thalassemia after MAC, published by Lucarelli et al. [34], no secondary graft failure was seen in patients achieving CC at 2 months post-HSCT, whereas 35 out of the 108 patients with MC at 2 months post-HSCT did develop graft failure. The risk of secondary graft failure was correlated with the degree of chimerism at day 60 . Ninety percent of the patients with a MC $<75 \%$ donor origin on peripheral blood went on to reject their graft versus only $13 \%$ of patients who had a donor chimerism of $>90 \%$ on peripheral blood $[34,36]$.

In a cohort of 91 patients, who received HSCT for severe aplastic anemia (SAA), no secondary graft failure was observed in patients who achieved CC immediately after HSCT (at day 30) or who showed an increasing donor chimerism post-HSCT and who achieved CC within 1 year post transplant. However, a decrease in donor chimerism of $\geq 15 \%$ on peripheral blood during followup was a significant risk factor for secondary graft failure, with 10/14 patients (71\%) going on to experience secondary graft failure during further follow-up (median time to graft failure not reported). In the majority of patients, this decreasing donor chimerism of $\geq 15 \%$ and subsequent graft failure $(7 / 10)$ occurred during the tapering of immunosuppressive therapy [35].

In a smaller patient cohort, consisting of 34 children transplanted for SAA in Germany, 4/34 patients presented with a donor chimerism of $<70 \%$ during follow-up. Two of these patients went on to experience secondary graft failure requiring a second HSCT. In light of these results the remaining 2 patients received $D L I$ infusion to prevent graft failure [37].

\section{Chimerism analysis and risk of relapse in malignant disease}

Relapse prediction using chimerism relies on the interpretation of chimerism kinetics. A sustained MC or decreasing donor chimerism early after HSCT has been shown to be an independent risk factor for relapse and impaired survival after MAC, RIC or NMA conditioning, in both adults and children, independent of the underlying hematological malignancy $[9,25-27,31,32,38-40]$.

The largest described adult cohort to date, which consisted of 688 retrospectively analysed patients who received T-cell replete RIC transplants for hematological malignancies, showed that peripheral blood donor chimerism of $<90 \%$ at day 30 and day 100 was independently associated with higher relapse rate and lower Progression free survival (PFS). A donor chimerism $<90 \%$ at day 100 was also independently associated with impaired Overall survival (OS). Day 100 chimerism was the best predictor of outcome [25].

The time needed to reach $\mathrm{CC}$ was also shown to be associated with the probability of relapse $[32,38,41]$. Chen et al. [38] showed that early achievement of complete chimerism in pediatric ALL patients after MAC transplant (median 15 days) was associated with a longer PFS. Similarly, Lassaletta et al. [41] showed that the probability of relapse in patients with hematological malignancies was significantly lower in patients who reached a CC by day 30 .

In patients with a disease characterized by a specific measurable residual disease (MRD) marker, early detection of relapse is primarily based on MRD detection by molecular biology or immunophenotype. However, as these disease markers can be lost on the occasion of disease relapse, a decreasing donor chimerism can be the first sign of disease relapse. It is also important to remember that up to $50 \%$ of the transplant population does not have a specific MRD marker that can be used in 
follow up $[9,42]$. The use of repetitive chimerism analysis, therefore, remains a necessary part of posttransplant follow-up, especially in the case of RIC or NMA transplants $[1,9]$.

Additionally, chimerism analysis can be used to confirm the presence of donor cell-derived leukemia. In this case, leukemia relapse occurs post-HSCT without decrease in donor chimerism, i.e. in patients with CC $[43,44]$. The use of FISH or cytogenetics for chimerism analysis in sex mismatched HSCT in this indication is limited, as loss of sex chromosomes with age as well as in hematological malignancies has been clearly described [44-46].

There is no general consensus on the ideal regimen of post-HSCT chimerism follow-up. Intervals ranging from weekly to monthly chimerism analysis have been used in clinical trials. We want to stress that it is primarily the dynamics or kinetics of a patient's chimerism status, which can only be determined by serial analysis at regular intervals, that will allow for the interpretation of the risk of (impending) relapse or secondary graft failure [9,24-26].

Based on the trials available and taking into account the practicality of these regimens in a 'real world' setting, we would propose that chimerism analysis on unfractionated peripheral blood and T-cells should ideally be performed at 1, 3, 6 and 12 months post transplant in case of MAC transplantation and at 1, 2, 3, 6 and 12 months post-HSCT in case of RIC or NMA transplant, in order to adequately interpret the underlying kinetics. In case of CC in MAC transplants follow-up can be limited. Further analysis is recommended at least once a year up to a minimum of 5 years post-HSCT to monitor for secondary graft failure and as a marker of possible minimal residual disease and relapse. In patients without a MRD marker chimerism analysis should be considered as part of routine disease surveillance in follow-up [24-26,28,29,31,32,35-38,41].

As the time frame between changes in donor chimerism and occurrence of relapse is unpredictable (ranging from 1 to several weeks [32]), serial analyses should be performed at short time intervals when a decreasing donor chimerism or persistent $M C$ is detected. Taking into account the required turnaround time of results as well as the short time interval observed between occurrence of a decreasing donor chimerism and occurrence of relapse, chimerism analysis performed every 2 weeks on peripheral blood samples are considered feasible. Laboratory turnaround time of samples should therefore be reduced to a minimum in these circumstances and ideally be limited to a maximum of 5 days [2].

\section{Chimerism analysis in non-malignant disease}

Contrary to malignant hematological diseases, complete donor chimerism is not a prerequisite for cure in patients with non-malignant hematological diseases, such as hemoglobinopathies, primary immunodeficiencies and bone marrow failure syndromes, where a partial replacement of autologous hematopoiesis can result in adequate clinical outcomes. Achieving a state of stable MC can be sufficient to improve hematopoiesis, restore immunocompetence or replace the deficient enzyme in these patients $[1,4,47,48]$.

In order to reduce transplant-related morbidity and mortality, the use of RIC regimens is more common than in malignant disease. However, this results in a higher likelihood of MC post-HSCT and an increased risk of graft rejection and nonengraftment $[1,34,35,49]$.

Long-term stable MC without disease relapse or graft rejection has been described in a considerable number of patients after HSCT for nonmalignant disease and has been described as late as 13 years postHSCT in published series. In our personal experience, we have identified patients with lifelong stable $M C$ after HSCT for immunodeficiency syndromes [34,50]. Long-term follow-up studies have shown that fluctuations in chimerism level are mostly seen during the first 24 months post-HSCT, with more stable patterns emerging in later follow up [48,50]. A matched case-control analysis between patients transplanted for nonmalignant disease with a long-term stable $M C$ and patients with CC post-HSCT performed by Stikvoort et al. [48,50] at a median time of 10 years post-HSCT, showed that there was no difference in prognosis between both patient groups. Interestingly, in a study of 85 children who underwent HSCT for nonmalignant disease, Faraci et al. [51] found that OS at 3 years was higher in patients with stable MC compared to patients with CC.

The presence of a MC in itself is therefore not equivalent to disease relapse or impending secondary graft failure in patients transplanted for non-malignant disease. Identification of a stable mixed chimerism is an important feature of clinical follow-up requiring continuation of chimerism analysis for longer periods of time.

\section{Pre-emptive post-HSCT interventions based on chimerism analysis}

The reduction of immune suppression and use of donor lymphocyte infusions (DLI) can be used to convert a MC to CC, thereby boosting the GVT effect [5256]. Achievement of CC as result of these interventions has been shown to provide a survival benefit $[54,55]$.

In a prospective study of 81 pediatric patients undergoing HSCT for AML and ALL, DLI were administered post-HSCT if MC (defined as <99\% cells of donor origin on peripheral blood using rt-qPCR) was present on 2 consecutive weekly analyses. Thirty-eight patients received DLIs, with 33 of these patients showing an increase in donor chimerism and 3 patients converting 
to CC. More importantly, an increase in OS at 3 years was observed in patients achieving CC compared to those with partial or no response [54]. Additionally, a prospective study which included 71 pediatric patients transplanted for AML found that the rapid tapering of immune suppression and subsequent administration of DLIs in patients presenting with MC on 2 consecutive peripheral blood or bone marrow samples until achievement of CC could improve RFS [55].

Donor lymphocyte infusion has also been shown to improve chimerism in non-malignant diseases. In a retrospective analysis of 26 pediatric patients receiving DLI for MC post-HSCT for non-malignant disease, $56 \%$ of patients showed an improvement of their chimerism status, with conversion to CC in $37 \%$ [56].

\section{Cell source}

\section{Lineage-specific chimerism analysis on peripheral} blood samples

Chimerism analysis of specific white blood cell subsets can provide a higher sensitivity and specificity for the detection of recipient hematopoiesis allowing for an earlier and more accurate detection of possible relapse $[8,57,58]$. Cell populations as small as $1 \%$ of the total white blood cell count can be isolated from blood samples and used for chimerism analysis [2,8,57-59].

\section{CD34+ chimerism}

CD34 is expressed on the majority of blast cells in AML, MDS and ALL populations and can be used in in these patient populations as a more sensitive predictor of disease relapse compared to whole blood chimerism, especially in the absence of a disease-specific MRD marker [57-61]. Unnikrishnan et al. [60] were able to show that a CD34+ donor chimerism of $<80 \%$ could predict relapse with a sensitivity of $100 \%$ and specificity of $67 \%$, compared to $14 \%$ and $83 \%$ for donor chimerism on whole blood (based on a population of 13 patients, 7 of which relapsed post-HSCT). Similarly, Hoffman et al. [61] showed that a CD34+ donor chimerism of $<80 \%$ had a $100 \%$ sensitivity for prediction of relapse. In a study carried out in 35 relapsed postHSCT patients treated with MAC or RIC by Bornhauser et al. [59], a CD34+ donor chimerism of $<80 \%$ had a positive predictive value for relapse of $80 \%$ and was significantly associated with a decreased OS and PFS at 4 years.

Based on the available evidence, a decline in CD34 + donor chimerism occurs earlier prior to relapse compared to a decline in peripheral whole blood chimerism [58-61]. In the patients studied by Urrikrishnan et al. [60] who showed hematological relapse, a decline of $\mathrm{CD} 34+$ donor chimerism could be documented at a median of 69 days prior to documentation of relapse (with a range of 4-175 days) and in the population studied by Thiede et al. [58] a decline in CD34+ donor chimerism could be detected 12-97 days prior to clinical relapse in $20 / 22$ patients.

\section{CD3 chimerism}

The presence of mixed T-cell chimerism has been linked to an increased relapse risk, inferior PFS and a higher graft rejection rate $[25,31,33,62,63]$. The use of T-cell donor chimerism, in addition to whole blood analysis, can provide a higher sensitivity for the detection of MC, as significant discrepancies between peripheral blood chimerism and $\mathrm{CD} 3+$ chimerism have been observed in about $10 \%$ of the HSCT population [32,64], most often occurring in MAC transplants [32]. In these patients, the presence of a MC can be missed if analysis is only performed on whole blood samples $[32,64]$.

In the cohort of 688 patients retrospectively analysed by Koreth et al. [25], a donor T-cell chimerism of $<70 \%$ at day 100 was associated with a higher relapse rate and an impaired PFS in multivariate analysis. However, use of T-cell chimerism did not provide a higher predictive capacity for PFS than day 100 whole blood chimerism in multivariate analysis.

Lee et al. [65] showed that patients transplanted for AML and MDS with a mixed T-cell chimerism of $\leq 85 \%$ between day 90 and 120 had a significantly lower PFS at 3 years post-transplant compared to patients with a T-cell donor chimerism of $>85 \%$. A comparison with whole blood chimerism, however, was not made. Also, in a retrospective analysis of 322 HSCT patients treated for hematological malignancies with a nonmyeloablative conditioning, performed by Baron et al. [62], the achievement of full donor T-cell chimerism ( $\geq 95 \%)$ was correlated with a significantly decreased risk of disease progression or disease relapse compared to patients with $<95 \%$ donor T-cell chimerism. Unfortunately, no data was presented on whole blood chimerism in these patients.

We would suggest using a combination of T-cell chimerism and whole blood chimerism in routine follow-up at day 30,60 and 90 in case of RIC transplant, in order to correctly interpret chimerism kinetics. Minimally it should be obtained at day 90-100 postHSCT and in cases presenting with a persistent MC or decreasing donor chimerism. In case of discrepancies between whole blood chimerism and T-cell chimerism, a combination of both tests should be used in further follow-up. A decreasing T-cell chimerism, in spite of stable whole blood chimerism, should prompt clinical action and decision-making.

\section{Other cell lineages}

Although other cell lineages such as CD19 + B cells and NK-cells have been used in clinical trials, there is 
currently not enough evidence to standardize their use in routine clinical follow-up [4,57].

\section{Bone marrow chimerism}

In addition to chimerism analysis on peripheral blood, bone marrow analysis can also be performed. The additional value of bone marrow chimerism in standard follow-up is not well established due to a lack of follow-up studies analyzing the role of bone marrow chimerism in relation to relapse or graft failure. It has been shown that the use of bone marrow for chimerism analysis in combination with peripheral blood analysis can increase the sensitivity of MC detection. However, it also results in a larger number of patients identified with MC who present with normal engraftment kinetics and who do not relapse post-HSCT $[9,18]$. This could be due to a contamination of bone marrow samples by autologous stromal cells $[9,18]$ and should be taken into account when interpreting results of bone marrow chimerism. Additionally, results of chimerism analysis in peripheral blood have been shown to be equivalent to results from analysis of bone marrow [66]. A study performed by Rauwerdink et al. [66] could show no difference in results between chimerism analysis performed on unfractionated peripheral blood and bone marrow in 42 patients at day 30, 60 and 90 post-HSCT.

\section{Clinical use of chimerism analysis: recommendations}

(1) After MAC, chimerism analysis on unfractionated peripheral blood is ideally performed at 1, 3, 6 and 12 months post-transplant. T-cell chimerism should ideally be performed at 3 months post-transplant. Analyses can be limited if CC has been reached. Further analysis is recommended at least once a year and should be considered as part of routine disease surveillance in patients without MRD marker.

(2) In case of RIC or NMA conditioning regimens, a sequential follow up with monitoring of chimerism kinetics is essential. Chimerism analysis on unfractionated peripheral blood should be performed at 1, 2, 3, 6 and 12 months postHSCT. Mixed chimerism with $<90 \%$ cells at day 30 and day 100 can be used to identify patients at high risk of relapse and impaired survival. Ideally, T-cell chimerism analysis should be obtained at 1, 2, 3 and 6 months post-HSCT or at the least at 3 months post-transplant and in the case of persistent MC or decreasing donor chimerism. Mixed T-cell chimerism <85-90\% can be used to identify patients at high risk of disease relapse and impaired survival. Further, follow up of chimerism analysis is recommended at least once a year for a minimum of 5 years postHSCT and should be considered as part of routine disease surveillance in patients without MRD marker. More frequent analysis is indicated when graft loss or rejection is suspected clinically, or in the event of a decreasing donor chimerism, at intervals of 2 weeks to 1 month.

(3) If available, MRD markers should be used for monitoring of relapse. Chimerism can be used to monitor disease relapse when no specific MRD markers are available. Combination of chimerism and follow-up of known MRD markers may provide additional information in the case of loss of a specific MRD marker on relapse.

(4) CD34+ chimerism on sorted peripheral blood cells, when available, can be used as an alternative to specific MRD markers in AML, MDS and ALL if leukemic blasts are known to express CD34.

(5) Bone marrow chimerism analysis can be used in addition to or instead of peripheral whole blood and lineage-specific chimerism analysis. Chimerism analysis on bone marrow can be considered when bone marrow aspirate is performed in routine clinical follow-up.

(6) Following therapeutic intervention sequential follow up of chimerism on unfractionated peripheral blood every 2-4 weeks is indicated. Addition of T-cell chimerism can be considered as useful. If stable CC is reached, analyses can be performed every 3-6 months.

(7) Following HSCT for non-malignant disorders (other than aplastic anemia) chimerism analysis should be performed at 1, 2 and 3 months postHSCT. If CC has been reached further follow-up is indicated when there is a clinical suspicion of rejection or relapse. In case of persistent $M C$, further follow-up at 2-3 monthly intervals is indicated until 24 months post-HSCT. In patients transplanted for aplastic anemia longer followup of chimerism is recommended, including during tapering of immune suppression.

\section{Current practice in Belgium}

There is at present no routine or standard practice used in Belgium when it comes to monitoring chimerism post-HSCT. A survey undertaken amongst 12 out of the 13 transplant centres in Belgium in 2019 showed that routine chimerism analysis was part of standard patient follow-up after allogeneic transplantation in $11 / 12$ centres.

All centres performing chimerism analysis use whole blood chimerism analysis as part of their routine follow-up. In 6/11 centres whole blood, bone marrow and CD3 chimerism are routinely performed, whereas in 4/11 centres whole blood and whole bone marrow 
are used (with CD3 chimerism available on indication) and in 1/11 centres only whole blood is used for routine follow-up. CD34+ bone marrow chimerism analysis has been used in several centres but only in specific cases and never in routine follow-up. Although CD34 + chimerism analysis on peripheral blood samples has been frequently used in clinical trials it is not currently performed in Belgium [59-61].

Current reimbursement criteria in Belgium allow for a maximum of six chimerism analyses on either whole blood, bone marrow or T-cells in the first year after transplant and a maximum of four analyses per year in the subsequent 4 years. This allows for only a minimal follow up of chimerism at the present time, without allowing for the eventuality of follow-up of split chimerism nor for follow-up of chimerism after a possible postHSCT intervention.

\section{Conclusion}

Serial chimerism analyses are an indispensible part of post-allogeneic HSCT follow-up, as there is clear evidence that the degree of chimerism and especially the observed chimerism kinetics are closely related to the risk of disease relapse and graft rejection. Close postHSCT follow-up of chimerism by repeated analyses will allow for the identification of patients with an indication for post-HSCT therapeutic interventions and their early application. The suggested frequency of followup of a patient's degree of chimerism, both in the immediate post-transplant setting and following any post-transplant therapeutic intervention is therefore of paramount importance to provide optimalpersonalized medicine, aiming at an increased survival post-allogeneic HSCT. Unfortunately, current reimbursement criteria do not allow for optimal patient followup. We would therefore urge the Health Authorities to revise these criteria in the light of these new guidelines, in order to provide adequate patient care.

\section{Disclosure statement}

No potential conflict of interest was reported by the authors.

[1] Bader P, Niethammer D, Willasch A, et al. How and when should we monitor chimerism after allogeneic stem cell transplantation? Bone Marrow Transplant. 2005 Jan;35(2):107-119. Review.

[2] Clark JR, Scott SD, Jack AL, et al. United Kingdom national external quality assessment service for leucocyte immunophenotyping chimerism working group. Monitoring of chimerism following allogeneic haematopoietic stem cell transplantation (HSCT): technical recommendations for the use of short tandem repeat (STR) based techniques, on behalf of the United Kingdom National External Quality Assessment Service for Leucocyte Immunophenotyping Chimerism Working Group. Br J Haematol. 2015 Jan;168(1):26-37. .

[3] Sellmann L, Rabe K, Bünting I, et al. Diagnostic value of highly-sensitive chimerism analysis after allogeneic stem cell transplantation. Bone Marrow Transplant. 2018 Nov;53(11):1457-1465. .

[4] Bader P. Documentation of engraftment and chimerism after HSCT. In: Carreras E, Dufour C, Mohty M, et al., editors. The EBMT Handbook: hematopoietic stem cell transplantation and cellular therapies. Cham (Switserland): Springer Open; 2019. p. 143-147.

[5] Miura Y, Tanaka J, Toubai T, et al. Analysis of donor-type chimerism in lineage-specific cell populations after allogeneic myeloablative and non-myeloablative stem cell transplantation. Bone Marrow Transplant. 2006 May;37(9):837-843. .

[6] Valcárcel D, Sureda A. Graft Failure. In: Carreras E, Dufour C, Mohty $M$, et al., editors. The EBMT Handbook: hematopoietic stem cell transplantation and cellular therapies. Cham (Switserland): Springer Open; 2019. p. 143-147.

[7] Kröger N, Bacher $\mathrm{U}$, Bader $\mathrm{P}$, et al. $\mathrm{NCl}$ first international workshop on the biology, prevention, and treatment of relapse after allogeneic hematopoietic stem cell transplantation: report from the committee on disease-specific methods and strategies for monitoring relapse following allogeneic stem cell transplantation. part II: chronic leukemias, myeloproliferative neoplasms, and lymphoid malignancies. Biol Blood Marrow Transplant. 2010 Oct;16(10):1325-1346.

[8] Lion T, Watzinger F, Preuner S, et al. The EuroChimerism concept for a standardized approach to chimerism analysis after allogeneic stem cell transplantation. Leukemia. 2012 Aug;26(8):1821-1828. .

[9] Ahci M, Stempelmann K, Buttkereit U, et al. Clinical utility of quantitative PCR for chimerism and engraftment monitoring after allogeneic stem cell transplantation for hematologic malignancies. Biol Blood Marrow Transplant. 2017 Oct;23(10):1658-1668.

[10] Khan F, Agarwal A, Agrawal S. Significance of chimerism in hematopoietic stem cell transplantation: new variations on an old theme. Bone Marrow Transplant. 2004 Jul;34(1):1-12. Review.

[11] Acquaviva C, Duval M, Mirebeau D, et al. Quantitative analysis of chimerism after allogeneic stem cell transplantation by PCR amplification of microsatellite markers and capillary electrophoresis with fluorescence detection: the Paris-Robert Debré experience. Leukemia. 2003 Jan;17(1):241-246.

[12] Chia WC, Khoo TS, SFS AW, et al. Multiplex STR panel for assessment of chimerism following hematopoietic stem cell transplantation (HSCT). Ann Hematol. 2019 May;98(5):1279-1291.

[13] Schraml $E$, Daxberger $H$, Watzinger $F$, et al. Quantitative analysis of chimerism after allogeneic stem cell transplantation by PCR amplification of microsatellite markers and capillary electrophoresis with fluorescence detection: the Vienna experience. Leukemia. 2003 Jan;17(1):224-227. Review.

[14] Alizadeh M, Bernard M, Danic B, et al. Quantitative assessment of hematopoietic chimerism after bone marrow transplantation by real-time quantitative polymerase chain reaction. Blood. 2002 Jun 15;99 (12):4618-4625.

[15] Frankfurt O, Zitzner JR, Tambur AR. Real-time qPCR for chimerism assessment in allogeneic hematopoietic stem cell transplants from unrelated adult and double umbilical cord blood. Hum Immunol. 2015 Mar;76 (2-3):155-160.

[16] Kletzel M, Huang W, Olszewski M, et al. Validation of chimerism in pediatric recipients of allogeneic 
hematopoietic stem cell transplantation (HSCT) a comparison between two methods: real-time PCR (qPCR) vs. variable number tandem repeats $P C R$ (VNTR PCR). Chimerism. 2013 Jan-Mar;4(1):1-8. .

[17] Kim SY, Jeong MH, Park N, et al. Chimerism monitoring after allogeneic hematopoietic stem cell transplantation using quantitative real-time PCR of biallelic insertion/deletion polymorphisms. J Mol Diagn. 2014 Nov;16(6):679-688.

[18] Willasch AM, Kreyenberg $H$, Shayegi $N$, et al. Monitoring of hematopoietic chimerism after transplantation for pediatric myelodysplastic syndrome: real-time or conventional short tandem repeat PCR in peripheral blood or bone marrow? Biol Blood Marrow Transplant. 2014 Dec;20(12):1918-1925.

[19] Koldehoff M, Steckel NK, Hlinka M, et al. Quantitative analysis of chimerism after allogeneic stem cell transplantation by real-time polymerase chain reaction with single nucleotide polymorphisms, standard tandem repeats, and Y-chromosome-specific sequences. Am J Hematol. 2006 Oct;81(10):735-746.

[20] Stahl T, Böhme MU, Kröger N, et al. Digital PCR to assess hematopoietic chimerism after allogeneic stem cell transplantation. Exp Hematol. 2015 Jun;43 (6):462-468.

[21] Thiede C, Bornhäuser M, Ehninger G. Strategies and clinical implications of chimerism diagnostics after allogeneic hematopoietic stem cell transplantation. Acta Haematol. 2004;112(1-2):16-23.

[22] Davis BH, Dasgupta A, Kussick S, et al. ICSH/ICCS Working Group. Validation of cell-based fluorescence assays: practice guidelines from the ICSH and ICCS part II - preanalytical issues. Cytometry B Clin Cytom. 2013 Sep-Oct;84(5):286-290.

[23] Borill V, Schlaphoff T, Du Toit E, et al. The use of short tandem repeat polymorphisms for monitoring chimerism following bone marrow transplantation: a short report. Hematology. 2008 Aug;13(4):210-214.

[24] Baron F, Baker JE, Storb R, et al. Kinetics of engraftment in patients with hematologic malignancies given allogeneic hematopoietic cell transplantation after nonmyeloablative conditioning. Blood. 2004 Oct 15;104 (8):2254-2262.

[25] Koreth J, Kim HT, Nikiforow S, et al. Donor chimerism early after reduced-intensity conditioning hematopoietic stem cell transplantation predicts relapse and survival. Biol Blood Marrow Transplant. 2014 Oct;20 (10):1516-1521.

[26] Reshef R, Hexner EO, Loren AW, et al. Early donor chimerism levels predict relapse and survival after allogeneic stem cell transplantation with reduced-intensity conditioning. Biol Blood Marrow Transplant. 2014 Nov;20(11):1758-1766.

[27] Storb R, Sandmaier BM. Nonmyeloablative allogeneic hematopoietic cell transplantation. Haematologica. 2016 May;101(5):521-530.

[28] Baron F, Little MT, Storb R. Kinetics of engraftment following allogeneic hematopoietic cell transplantation with reduced-intensity or nonmyeloablative conditioning. Blood Rev. 2005 May;19(3):153-164.

[29] Lee KH, Lee JH, Choi SJ, et al. Monthly prospective analysis of hematopoietic chimerism after allogeneic hematopoietic cell transplantation. Bone Marrow Transplant. 2003 Aug;32(4):423-431.

[30] Childs R, Clave E, Contentin N, et al. Engraftment kinetics after nonmyeloablative allogeneic peripheral blood stem cell transplantation: full donor T-cell chimerism precedes alloimmune responses. Blood. 1999 Nov 1;94(9):3234-3241.

[31] Thompson PA, Stingo F, Keating MJ, et al. Long-term follow-up of patients receiving allogeneic stem cell transplant for chronic lymphocytic leukaemia: mixed T-cell chimerism is associated with high relapse risk and inferior survival. Br J Haematol. 2017 May;177(4):567-577.

[32] Huisman C, de Weger RA, de Vries L, et al. Chimerism analysis within 6 months of allogeneic stem cell transplantation predicts relapse in acute myeloid leukemia. Bone Marrow Transplant. 2007 Mar;39(5):285-291. Epub 2007 Jan 29

[33] Breuer S, Preuner S, Fritsch G, et al. Early recipient chimerism testing in the T- and NK-cell lineages for risk assessment of graft rejection in pediatric patients undergoing allogeneic stem cell transplantation. Leukemia. 2012 Mar;26(3):509-519.

[34] Lucarelli G, Andreani M, Angelucci E. The cure of thalassemia by bone marrow transplantation. Blood Rev. 2002 Jun;16(2):81-85.

[35] Severe Aplastic Anaemia Working Party of the European Blood and Marrow Transplant Group, Lawler M, McCann SR, Marsh JC, et al. Serial chimerism analyses indicate that mixed haemopoietic chimerism influences the probability of graft rejection and disease recurrence following allogeneic stem cell transplantation (SCT) for severe aplastic anaemia (SAA): indication for routine assessment of chimerism post SCT for SAA. Br J Haematol. 2009 Mar;144(6):933-945.

[36] Gaziev J, Lucarelli G. Stem cell transplantation for hemoglobinopathies. Curr Opin Pediatr. 2003 Feb;15 (1):24-31.

[37] Hoelle W, Beck JF, Dueckers G, et al. Clinical relevance of serial quantitative analysis of hematopoietic chimerism after allogeneic stem cell transplantation in children for severe aplastic anemia. Bone Marrow Transplant. 2004 Jan;33(2):219-223.

[38] Chen CT, Gau JP, Liu JH, et al. Early achievement of full donor chimerism after allogeneic hematopoietic stem cell transplantation predicts lower relapse risk in patients with acute lymphoblastic leukemia. J Chin Med Assoc. 2018 Dec;81(12):1038-1043.

[39] Wiedemann B, Klyuchnikov E, Kröger N, et al. Chimerism studies with quantitative real-time PCR in stem cell recipients with acute myeloid leukemia. Exp Hematol. 2010 Dec;38(12):1261-1271.

[40] Gabr H, Youssry I, El-Ansary Y, et al. Chimerism in pediatric hematopoietic stem cell transplantation and its correlation with the clinical outcome. Transpl Immunol. 2017 Dec;45:53-58.

[41] Lassaletta A, Ramírez M, Montero JM, et al. Full donor chimerism by day 30 after allogeneic peripheral blood progenitor cell transplantation is associated with a low risk of relapse in pediatric patients with hematological malignancies. Leukemia. 2005 Apr;19(4):504-506.

[42] Kern W, Haferlach C, Haferlach T, et al. Monitoring of minimal residual disease in acute myeloid leukemia. Cancer. 2008 Jan 1;112(1):4-16.

[43] Hertenstein B, Hambach L, Bacigalupo A, et al. Development of leukemia in donor cells after allogeneic stem cell transplantation-a survey of the European Group for Blood and Marrow Transplantation (EBMT). Haematologica. 2005 Jul;90(7):969-975.

[44] Wiseman DH. Donor cell leukemia: a review. Biol Blood Marrow Transplant. 2011;17(6):771-789.

[45] Herens C, Brasseur E, Jamar M, et al. Loss of the Y chromosome in bone marrow cells: results on 1907 
consecutive cases of leukaemia and preleukaemia. Clin Lab Haematol. 1999 Feb;21(1):17-20.

[46] Stone JF, Sandberg AA. Sex chromosome aneuploidy and aging. Mutat Res. 1995 Oct;338(1-6):107-113.

[47] Abraham A, Hsieh $M$, Eapen $M$, et al. Relationship between mixed donor-recipient chimerism and disease recurrence after hematopoietic cell transplantation for sickle cell disease. Biol Blood Marrow Transplant. 2017 Dec;23(12):2178-2183.

[48] Stikvoort A, Sundin M, Uzunel M, et al. Long-term stable mixed chimerism after hematopoietic stem cell transplantation in patients with non-malignant disease, shall we be tolerant? PLoS One. 2016 May 6;11 (5):e0154737.

[49] Willasch A, Hoelle W, Kreyenberg $\mathrm{H}$, et al. Outcome of allogeneic stem cell transplantation in children with non-malignant diseases. Haematologica. 2006 Jun;91 (6):788-794.

[50] Stikvoort A, Gertow J, Sundin M, et al. Chimerism patterns of long-term stable mixed chimeras posthematopoietic stem cell transplantation in patients with nonmalignant diseases: follow-up of long-term stable mixed chimerism patients. Biol Blood Marrow Transplant. 2013 May;19(5):83844.

[51] Faraci $M$, Bagnasco $F$, Leoni $M$, et al. Evaluation of chimerism dynamics after allogeneic hematopoietic stem cell transplantation in children with nonmalignant diseases. Biol Blood Marrow Transplant. 2018 May;24(5):1088-1093.

[52] Caldemeyer LE, Akard LP, Edwards JR, et al. Donor lymphocyte infusions used to treat mixed-chimeric and high-risk patient populations in the relapsed and nonrelapsed settings after allogeneic transplantation for hematologic malignancies are associated with high five-year survival if persistent full donor chimerism is obtained or maintained. Biol Blood Marrow Transplant. 2017 Nov;23(11):1989-1997.

[53] Alyea EP, DeAngelo DJ, Moldrem J, et al. NCl First international workshop on the biology, prevention and treatment of relapse after allogeneic hematopoietic cell transplantation: report from the committee on prevention of relapse following allogeneic cell transplantation for hematologic malignancies. Biol Blood Marrow Transplant. 2010 Aug;16(8):1037-1069.

[54] Rujkijyanont P, Morris C, Kang G, et al. Risk-adapted donor lymphocyte infusion based on chimerism and donor source in pediatric leukemia. Blood Cancer J. 2013 Aug;30(3):e137.

[55] Rettinger E, Willasch AM, Kreyenberg $\mathrm{H}$, et al. Preemptive immunotherapy in childhood acute myeloid leukemia for patients showing evidence of mixed chimerism after allogeneic stem cell transplantation. Blood. 2011 Nov 17;118(20):5681-5688. Epub 2011 Sep 26.
[56] Haines HL, Bleesing JJ, Davies SM, et al. Outcomes of donor lymphocyte infusion for treatment of mixed donor chimerism after a reduced-intensity preparative regimen for pediatric patients with nonmalignant diseases. Biol Blood Marrow Transplant. 2015 Feb;21 (2):288-292.

[57] Preuner S, Peters C, Pötschger U, et al. Risk assessment of relapse by lineage-specific monitoring of chimerism in children undergoing allogeneic stem cell transplantation for acute fpl leukemia. Haematologica. 2016 Jun;101(6):741-746.

[58] Thiede C, Lutterbeck K, Oelschlägel U, et al. Detection of relapse by sequential monitoring of chimerism in circulating CD34+ cells. Ann Hematol. 2002;81(Suppl 2):S27-8.

[59] Bornhäuser $M$, Oelschlaegel $U$, Platzbecker $U$, et al. Monitoring of donor chimerism in sorted CD34+ peripheral blood cells allows the sensitive detection of imminent relapse after allogeneic stem cell transplantation. Haematologica. 2009 Nov;94 (11):1613-1617.

[60] Unnikrishnan A, Meacham AM, Goldstein SS, et al. CD34+ chimerism analysis for minimal residual disease monitoring after allogeneic hematopoietic cell transplantation. Leuk Res. 2018 Nov;74:110-112.

[61] Hoffmann JC, Stabla K, Burchert A, et al. Monitoring of acute myeloid leukemia patients after allogeneic stem cell transplantation employing semi-automated CD34 + donor cell chimerism analysis. Ann Hematol. 2014 Feb;93(2):279-285.

[62] Baron F, Maris MB, Sandmaier BM, et al. Graft-versustumor effects after allogeneic hematopoietic cell transplantation with nonmyeloablative conditioning. J Clin Oncol. 2005 Mar 20;23(9):1993-2003.

[63] Broglie L, Helenowski I, Jennings L, et al. Early mixed T-cell chimerism is predictive of pediatric AML or MDS relapse after hematopoietic stem cell transplant. Pediatr Blood Cancer. 2017 Sep;64(9).

[64] Pichler H, Fritsch G, König $M$, et al. Peripheral blood late mixed chimerism in leucocyte subpopulations following allogeneic stem cell transplantation for childhood malignancies: does it matter? $\mathrm{Br} J$ Haematol. 2016 Jun;173(6):905-917.

[65] Lee HC, Saliba RM, Rondon G, et al. Mixed T lymphocyte chimerism after allogeneic hematopoietic transplantation is predictive for relapse of acute myeloid leukemia and myelodysplastic syndromes. Biol Blood Marrow Transplant. 2015 Nov;21(11):1948-1954.

[66] Rauwerdink CA, Tsongalis GJ, Tosteson TD, et al. The practical application of chimerism analyses in allogeneic stem cell transplant recipients: blood chimerism is equivalent to marrow chimerism. Exp Mol Pathol. 2012 Dec;93(3):339-344. 